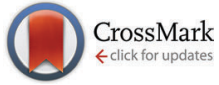

Cite this: J. Mater. Chem. B, 2015, 3, 3054

Received 11th January 2015,

Accepted 25th February 2015

DOI: $10.1039 / c 5 t b 00058 k$

www.rsc.org/MaterialsB

\section{Silica capsules enclosing P123 triblock copolymer micelles for flurbiprofen storage and release $\dagger$}

\author{
Stef Kerkhofs, ${ }^{a}$ Fadila Saïdi, ${ }^{b}$ Niels Vandervoort, ${ }^{a}$ Guy Van den Mooter, ${ }^{c}$ \\ Charlotte Martineau, ${ }^{\mathrm{b}}$ Francis Taulelle ${ }^{\mathrm{ab}}$ and Johan A. Martens ${ }^{{ }^{\mathrm{a}}}$
}

\begin{abstract}
Flurbiprofen was incorporated in 200-400 nm silica capsules filled with Pluronic P123 polyethylene oxide-polypropylene oxide-polyethylene oxide triblock copolymer. The assembly process of the capsules and the molecular organization of drug molecule, surfactant and silica were investigated using SAXS, TGA, SEM, DLS, DSC, ${ }^{13} \mathrm{C}$ single-pulse, CPMAS and ${ }^{1} \mathrm{H}-{ }^{1} \mathrm{H}$ two-dimensional NMR. Flurbiprofen molecules are molecularly dispersed inside polypropylene cores of P123 surfactant micelles occluded in a 20-30 nm thick silica shell. Flurbiprofen molecules in polypropylene cores of P123 micelles exhibit high mobility and are easily released after introduction in simulated gastrointestinal fluid and the solubility limit is reached within minutes. Release rates are favored at high $\mathrm{pH}$ due to acid dissociation of the carboxylic acid group of the flurbiprofen molecule. The molecular environment of flurbiprofen in these P123 filled silica capsules is different from ordered mesoporous silica materials synthesized using P123 as sacrificial template having the drug molecules adsorbed on the silica surface of pore walls. These findings uncover a new concept of storage and release of hydrophobic bioactive molecules.
\end{abstract}

\section{Introduction}

Pharmaceutical formulation of hydrophobic active compounds with low aqueous solubility in gastrointestinal (GI) fluids is very challenging, and the number of poorly soluble drug candidates facing this problem is steadily increasing. ${ }^{1-4}$ Poor aqueous solubility leads to low bioavailability of an oral drug due to limited uptake along the GI tract. ${ }^{2}$ Formulation with silica, and especially the ordered mesoporous silica (OMS) can offer a solution. Drug molecules stored in OMS pore walls are released upon contact with aqueous medium and generate a pH-independent supersaturated solution favouring transepithelial transport. ${ }^{2}$ OMS is a convenient carrier material because of its chemical stability, inertness, biocompatibility and ease of fuctionalization. ${ }^{5-7}$ OMS combines high surface area and large pore volume with uniform pores that can be finetuned in diameter from 2 to $50 \mathrm{~nm}$. OMS is synthesized using sacrificial supramolecular organic template, which is removed through calcination. Drug molecules are loaded in the pores via an impregnation or adsorption procedure involving the use of an organic solvent. ${ }^{2,3,6,7}$ A more recent development is to

\footnotetext{
${ }^{a}$ Centre for Surface Chemistry and Catalysis, KU Leuven, Heverlee, Belgium.

E-mail: Johan.Martens@biw.kuleuven.be

${ }^{b}$ Tectospin, Institut Lavoisier, Université de Versailles St Quentin en Yvelines, France

${ }^{c}$ Drug Delivery and Disposition, KU Leuven, Leuven, Belgium

$\dagger$ Electronic supplementary information (ESI) available. See DOI: 10.1039/ c5tb00058k
}

incorporate the active compound during the synthesis of the OMS carrier material. ${ }^{3,5-9}$ In such synthesis process, the active compound is solubilized in aqueous medium using an amphiphilic surfactant, which together with the drug serves as the supramolecular template for the mesoscopic ordering of the silica. Some reported formulations are based on cetyltrimethylammonimum (CTAB) surfactant. ${ }^{3,6,9,10}$ The concept of active compound release from OMS-embedded CTAB micelles has been extended to nutraceuticals. ${ }^{9}$ The use of CTAB has some drawbacks, however. It is considered toxic and has been reported to present cytotoxicity comparable to some cancer drugs. ${ }^{7,11}$ The use of non toxic templates such as anionic surfactants, ${ }^{3}$ alkyl maltosides $^{5}$ and triblock copolymer poloxamer ${ }^{7}$ surfactants have been investigated. Generally, organic solvent is needed to dissolve the drug molecules in the synthesis medium and to achieve incorporation in the surfactant-silica composite. ${ }^{3,5}$

Here we report the synthesis of surfactant containing capsules with high drug loading capacity. We discovered this material in an attempt to prepare a flurbiprofen loaded COK-12, which is an OMS which can be synthesized using Pluronic P123 triblock copolymer surfactant at ambient temperature in citric acidcitrate buffered solution. ${ }^{11,12}$ Flurbiprofen is a nonsteroidal anti-inflammatory drug comparable to ibuprofen both in function as in chemical structure. ${ }^{13}$ It is administered in treatment of arthritis, migraine and inflammation. Flurbiprofen is a weakly acidic hydrophobic molecule, with a $\mathrm{p} K_{\mathrm{a}}$ of 4.2 , melting point of $117{ }^{\circ} \mathrm{C}$ and solubility of $8 \mathrm{mg} \mathrm{l}^{-1}$ in demineralized water. ${ }^{14-16}$ Owing to the acid dissociation of the carboxylic acid 
group the solubility is $\mathrm{pH}$ dependent, enabling $\mathrm{pH}$ control over the release from its formulations. ${ }^{14}$ Capsules filled with P123 surfactant and flurbiprofen assembled spontaneously in the synthesis mixture. The formation process and the molecular organization of flurbiprofen, P123 surfactant and silica was probed using DLS, SAXS, TGA, DSC and ${ }^{1} \mathrm{H}$ and ${ }^{13} \mathrm{C}$ solid-state $\mathrm{NMR}$, and in vitro release experiments.

\section{Experimental section}

\section{Synthesis}

Flurbiprofen-P123-silica capsule (FPS) was prepared by adapting the synthesis procedure for COK-12 performed at room temperature and slightly acidic pH. ${ }^{11,12}$ Standard buffered P123 surfactant solution was prepared by dissolving $4.0 \mathrm{~g}$ triblock copolymer Pluronic P123 (BASF, Belgium), $3.684 \mathrm{~g}$ citric acid monohydrate (Sigma Aldrich) and $2.540 \mathrm{~g}$ trisodium citrate dehydrate (Sigma Aldrich) in $107.5 \mathrm{~g}$ demineralized water. $600 \mathrm{mg}$ flurbiprofen (Sigma Aldrich) was added to $100 \mathrm{ml}$ of citrate buffered P123 surfactant solution. The solution was stirred until all flurbiprofen was solubilized and an opaque micellar solution was obtained. The initial $\mathrm{pH}$ of the citrate buffered, solution was 3.7. To this, $9.23 \mathrm{~g}$ sodium silicate solution (Merck, extra pure, $27 \% \mathrm{SiO}_{2}, 10 \%$ $\mathrm{NaOH}$ ), diluted in $26.64 \mathrm{~g}$ demineralized water was added while stirring vigorously. A white suspension was obtained instantaneously, which was aged under quiescent conditions for $24 \mathrm{~h}$. Solid powder was recovered by filtration, washed with demineralized water and dried at $50{ }^{\circ} \mathrm{C}$. The $\mathrm{pH}$ was measured using a Mettler Toledo, Inlab ${ }^{\mathbb{R}}$ Expert Pro $\mathrm{pH}$ electrode. The $\mathrm{pH}$ of the filtrate was 5.3. For the purpose of comparison, COK-12 was synthesized using the same procedure in absence of flurbiprofen.

\section{Materials characterization}

Dynamic Light Scattering (DLS) of P123 micellar solution was performed in polystyrene cuvettes on a 90Plus Particle Size Analyzer (Brookhaven) equipped with $659 \mathrm{~nm}$ laser, under a detection angle of $90^{\circ}$. Fluctuations in the scattered light intensity were correlated between $5 \mu$ s and 1 s. Correlation functions were analyzed with Igor Pro 6.2, using the Clementine package for modeling of decay kinetics based on the Maximum Entropy method. Decay times were converted to hydrodynamic diameters using the Stokes-Einstein equation, yielding intensity weighted particle size distributions. The viscosity was determined using a rolling ball viscometer (AMVn automated microviscometer, Anton Paar).

Small Angle X-ray Scattering (SAXS) patterns of powder samples were recorded at room temperature using a SAXSess $\mathrm{mc}^{2}$ instrument (Anton Paar) with rotating powder cell, linecollimated $\mathrm{Cu}_{\mathrm{K} \alpha}$ radiation and a $2 \mathrm{D}$ imaging plate detector in combination with a temperature controlled RotorCell sample stage. SAXS patterns were normalized to incident beam intensity. Background subtraction and desmearing were performed using SAXSquant software.

Nitrogen adsorption isotherms were recorded at $-196.8{ }^{\circ} \mathrm{C}$ on a Micromeritics Tristar 3000 apparatus (Micromeritics).
Samples were degassed at $100{ }^{\circ} \mathrm{C}$ for $12 \mathrm{~h}$ under nitrogen flow. Specific surface area was estimated using the BET model in the relative pressure range $\left(P / P_{0}\right)$ between 0.05 and 0.3 . Pore volumes were estimated using $t$-plot method.

Scanning Electron Microscopy (SEM) observations were made with a Nova NanoSEM450 (FEI) operated at $1.5 \mathrm{kV}$. Powder samples were dispersed on carbon tape and measured without applying a conductive coating.

Thermogravimetric Analysis (TGA) was performed on a TA Instruments TGA Q500 under oxidative atmosphere $\left(\mathrm{O}_{2}: \mathrm{N}_{2}\right.$ molar ratio of 9:1; flow rate of $100 \mathrm{ml} \mathrm{min}^{-1}$ ). Samples were heated to $850{ }^{\circ} \mathrm{C}$ at a heating rate of $5{ }^{\circ} \mathrm{C} \mathrm{min}^{-1}$.

Differential Scanning Calorimetry (DSC) was performed on a TA instruments DSC Q2000. Samples were analyzed in crimped aluminum pans, at temperatures from $-20{ }^{\circ} \mathrm{C}$ to $130{ }^{\circ} \mathrm{C}$ using a heating rate of $20{ }^{\circ} \mathrm{C} \mathrm{min}^{-1}$. Temperature measurement was calibrated and validated using indium and $n$-octadecane standards while enthalpy measurement was calibrated and validated using indium standards.

Solid-state NMR spectra were recorded on an Avance Bruker 500 NMR spectrometer (static magnetic field of $11.7 \mathrm{~T}$ ). The samples were packed in $4 \mathrm{~mm}$ outer diameter zirconia rotors and spun at $10 \mathrm{kHz} .{ }^{13} \mathrm{C}$ single pulse measurements were performed using a $5 \mu \mathrm{s}$ $90^{\circ}$ pulse, a short recycle delay of $4 \mathrm{~s}$ recycle (to select mobile species) and ${ }^{1} \mathrm{H}$ SPINAL-64 ${ }^{17}$ decoupling. ${ }^{1} \mathrm{H} \rightarrow{ }^{13} \mathrm{C}$ cross-polarization (CP-MAS) NMR spectra were recorded using a $3 \mu \mathrm{s} 90^{\circ}$ pulse for ${ }^{1} \mathrm{H}, 50 \mathrm{kHz}$ radio-frequency $(\mathrm{RF})$ field on ${ }^{13} \mathrm{C}$, and $4 \mathrm{~ms}$ contact time. ${ }^{1} \mathrm{H}$ SPINAL-64 decoupling was applied during the acquisition period. About 7000 transients were accumulated for each sample. The ${ }^{1} \mathrm{H}-{ }^{1} \mathrm{H}$ 2D single-quantum single-quantum (SQ-SQ) NMR spectra were recorded using the radio-frequency driven recoupling (RFDR) sequence with recoupling times of $4.5 \mathrm{~ms}$. The States procedure ${ }^{18}$ was applied to obtain phase sensitive spectra. The ${ }^{1} \mathrm{H}$ and ${ }^{13} \mathrm{C}$ chemical shift were referenced to tetramethylsilane (TMS) at $0 \mathrm{ppm}$. The Dmfit software was used for the data analysis. ${ }^{19}$

\section{Flurbiprofen release experiments}

FPS powder was suspended in $0.1 \mathrm{M}$ phosphate buffer ( $\mathrm{pH}$ 6.8) or $0.1 \mathrm{M} \mathrm{HCl}$ solution (pH 1.2). Typically, $288 \mathrm{mg}$ FPS powder was suspended in $240 \mathrm{ml}$ dissolution medium. Flurbiprofen release from the FPS suspension was performed under gentle agitation using a rotary mixer at room temperature $\left(25{ }^{\circ} \mathrm{C}\right)$. At specific time intervals, with a first sample taken after 3 minutes, $1.5 \mathrm{ml}$ samples were collected and filtered through $0.45 \mu \mathrm{m}$ PTFE membrane before analysis. After $4 \mathrm{~h}$ release, the remaining suspension was filtered to recover the solids for analysis.

To simulate release during the gastric-to-intestinal transition, $25 \mathrm{mg}$ of flurbiprofen formulation was added to $20 \mathrm{ml} \mathrm{HCl}$ $(0.1 \mathrm{M})$ solution. After $1 \mathrm{~h}, 180 \mathrm{ml}$ of $0.1 \mathrm{M}$ phosphate buffer solution was added. The suspension was sampled regularly to monitor flurbiprofen release.

Flurbiprofen concentration in solution was determined using HPLC-UV. The chromatographic system consisted of a LaChrom ${ }^{\circledR}$ L-7100 pump, a L-7200 autosampler, a UV-detector model L-7420 set at $248 \mathrm{~nm}$ and a D-7000 interface (All from Merck-Hitachi, Darmstadt, Germany). The column used was a 
Chromolith Performance RP-18e column (Merck-Hitachi, Darmstadt, Germany) and the mobile phase consisted of phosphate buffer (0.01 M, pH 5.5) and HPLC grade acetonitrile in a $70: 30 \mathrm{v} / \mathrm{v}$ ratio at a total flow rate of $1 \mathrm{ml} \mathrm{min}^{-1}$. Flurbiprofen UV signals were monitored and peaks were integrated using D-7000 software. Standard curves were linear over the concentration range of $1-300 \mathrm{mg} \mathrm{l}^{-1}$.

\section{Results and discussion}

\section{Synthesis of FPS}

Pluronic P123 with formula $(\mathrm{pEO})_{20}-(\mathrm{pPO})_{70}-(\mathrm{pEO})_{20}$ is a triblock copolymer consisting of a central hydrophobic polypropylene oxide (pPO) chain between two more hydrophilic polyethylene oxide (pEO) chains. P123 unimers in aqueous solution self-assemble to spherical micelles consisting of a hydrophobic core of pPO chains and an fuzzy layer of pEO, which stabilizes the micelles sterically. ${ }^{20}$ In citric acid-citrate buffered aqueous solution, P123 triblock copolymer self-assembled into spherical micelles measuring $\mathrm{ca}$. $14 \mathrm{~nm}$ according to DLS (Fig. 1 and Fig. S1, ESI $\dagger$ ), in agreement with literature. ${ }^{12,21,22}$ Hydrophobic compounds added to micellar P123 solution can be solubilized inside the hydrophobic pPO core of the micelles, causing swelling. ${ }^{14,20,23-25}$ A typical example is the addition of trimethyl benzene to provoke swelling of P123 micelles serving as supramolecular sacrificial templates for wide pore OMS materials synthesis. ${ }^{12,20,26-28}$ Pore diameters up to $30 \mathrm{~nm}$ have been reached using this approach. ${ }^{26,27}$ Loading Pluronic P123 with docetaxel (DTX), an antineoplastic anticancer drug, led to DTX swollen micelles. Spherically shaped micelles as large as $84 \mathrm{~nm}$ have been observed. ${ }^{29}$ Flurbiprofen loading into P123 micelles was performed at $\mathrm{pH} 3.7$ in order to prevent flurbiprofen from acidbase dissociation and to maximize flurbiprofen uptake in the micelles. ${ }^{14,23}$ Flurbiprofen addition to P123 micelle solution led to a large increase of micelle size to $c a .180 \mathrm{~nm}$ (Fig. 1). Drug-toPluronic ratio was high ( $c a .1: 6 \mathrm{w} / \mathrm{w}$ ), a considerable amount of flurbiprofen had to be incorporated inside the Pluronic micelles.

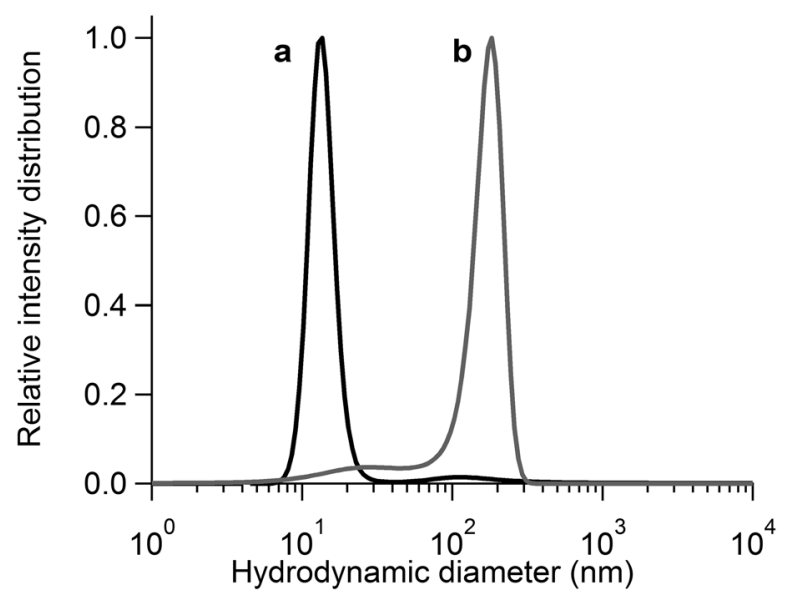

Fig. 1 Intensity weighted size distribution of P123 micelles in citrate buffered solution: (a) before flurbiprofen addition and (b) after flurbiprofen addition, determined by DLS.
The here observed micellar size of $180 \mathrm{~nm}$ could be the result of coalescence in addition to swelling.

Addition of sodium silicate to the buffered P123 solution containing flurbiprofen led to rapid precipitation of a white powder referred to as FPS (Flurbiprofen-P123-silica capsule). Flurbiprofen was concentrated in the FPS material. The flurbiprofen concentration in the filtrate was very low. Only about $1.5 \%$ of the initially added flurbiprofen was left in solution. The flurbiprofen incorporation efficiency was $98.5 \%$. The flurbiprofen content of FPS determined through solubilization in ethanol was $8.90 \mathrm{wt} \%$.

\section{Characterization of FPS}

TGA analysis of FPS material in oxidizing atmosphere showed a weight loss of roughly $60 \mathrm{wt} \%$ ascribed to P123 and flurbiprofen (Fig. S2, ESI $\dagger$ ). The presence of flurbiprofen and P123 in FPS material was confirmed by ${ }^{13} \mathrm{C}$ MAS NMR (Fig. 2a) showing the signals of flurbiprofen and P123. Solubilization of flurbiprofen inside Pluronic micelles was confirmed by ${ }^{1} \mathrm{H}-{ }^{1} \mathrm{H} 2 \mathrm{D}$
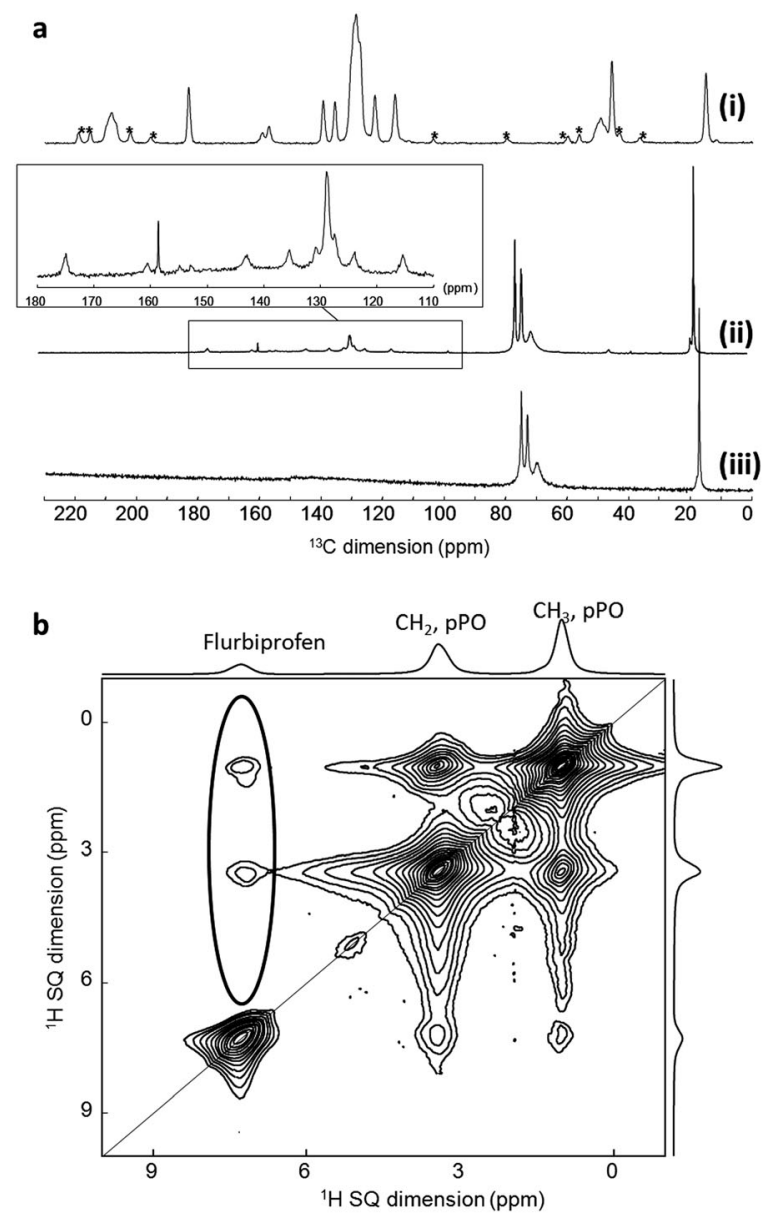

Fig. 2 (a) ${ }^{13} \mathrm{C}$ CPMAS NMR spectrum of flurbiprofen, stars indicating the position of spinning sidebands (i), ${ }^{13} \mathrm{C}$ MAS NMR spectra of FPS, showing both flurbiprofen and Pluronic P123 (ii) and of P123 in as-synthesized COK-12 reference material (iii). (b) $2 \mathrm{D}{ }^{1} \mathrm{H}-{ }^{1} \mathrm{H}$ SQ-SQ NMR correlation spectrum of FPS. The circled signals are cross-peaks between flurbiprofen protons and protons of pPO chains of P123. 
solid-state NMR (Fig. 2b). The 2D NMR revealed a close proximity between the protons of the flurbiprofen molecules and those of the pPO chains, being at a distance of only a few Ångströms in order to enable the coupling.

In SEM (Fig. 3a and b) FPS appeared as aggregated spherical particles with mean diameters of 200-400 $\mathrm{nm}$. Closer inspection of SEM images occasionally observed damaged spheres (Fig. 3c), revealing the hollow nature and the formation of submicron sized capsules with 20-30 nm shell thickness. The SAXS pattern of FPS (Fig. 4a) essentially showed only one broad diffraction peak revealing poor ordering. After removal of the P123 surfactant and flurbiprofen through calcination at $550{ }^{\circ} \mathrm{C}$, the material did not show diffraction peaks.

The porosity of calcined FPS was investigated using nitrogen adsorption (Fig. 5a). The nitrogen adsorption isotherm according to IUPAC classification corresponds to type IV, with an $\mathrm{H}_{2}$ type hysteresis loop. Such adsorption isotherm is characteristic for macropores (diameters larger than $50 \mathrm{~nm}$ ), which are filled through capillary condensation only at very high relative pressure. The strong hysteresis in the desorption isotherm and the sudden release at $P / P_{0}=0.45$ indicates the inner void can be accessed through narrow, less than $2 \mathrm{~nm}$ wide openings. According to $\mathrm{N}_{2}$ adsorption, the silica shell indeed is microporous. The micropore volume amounted to $0.164 \mathrm{~cm}^{3} \mathrm{~g}^{-1}$. The formation of micropores in silica materials synthesized using P123 surfactant
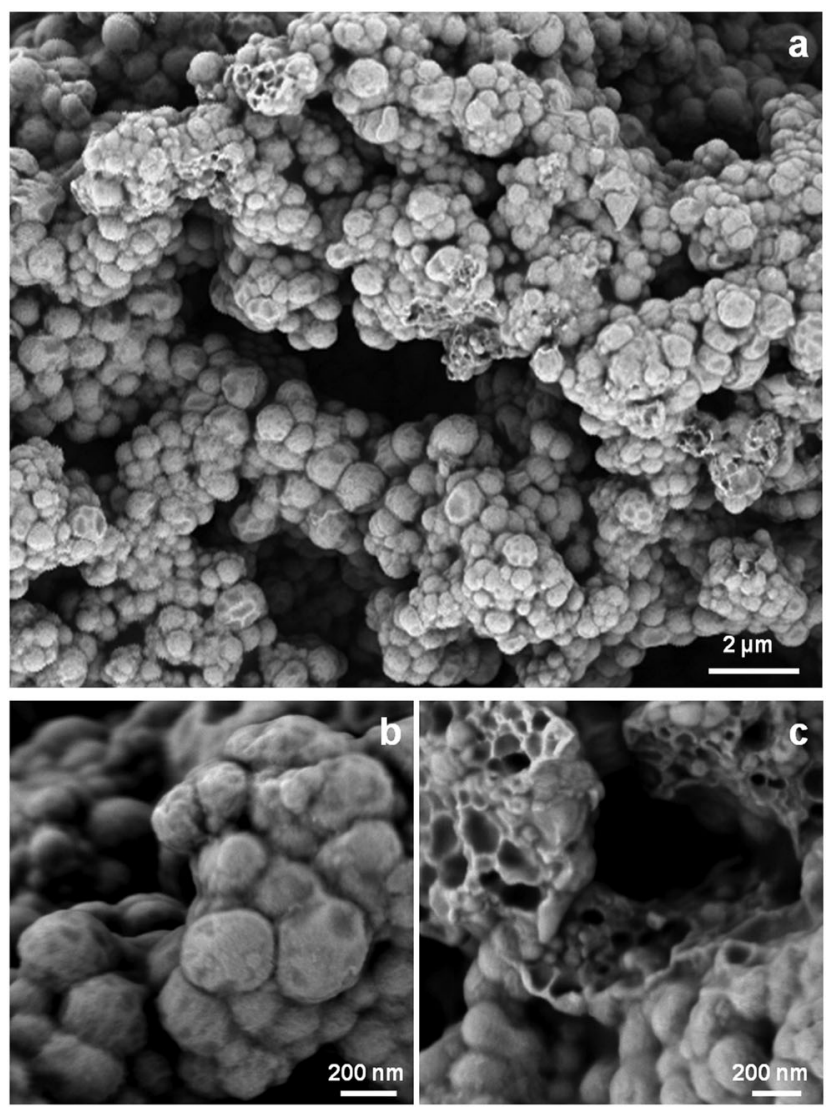

Fig. 3 Low magnification SEM image of FPS material (a). Higher magnifications show details of intact (b) and damaged capsules (c).

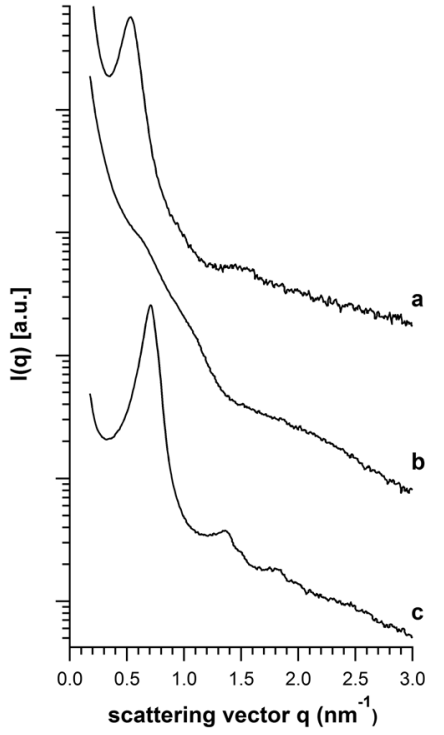

Fig. 4 Powder SAXS patterns of as-synthesized and calcined materials: (a) FPS; (b) calcined FPS; (c) calcined COK-12. Curves were shifted vertically for clarity.

has been ascribed to pEO moieties embedded in the silica which create micropores upon calcination. $\mathrm{N}_{2}$ adsorption behavior is consistent with the presence of hollow capsules observed with SEM (Fig. 3b).

These characterizations revealed FPS is different from COK-12, while the only difference in the synthesis procedure is the addition of flurbiprofen. FPS consists of $200-400 \mathrm{~nm}$ spherical capsules, with $20-30 \mathrm{~nm}$ thick silica shell occluding P123 and flurbiprofen. COK-12 is an OMS with hexagonal platelet morphology, roughly $250 \mathrm{~nm}$ thick and $500 \mu \mathrm{m}$ in diameter. ${ }^{11,12}$ It has $P 6 m$ hexagonal symmetry (Fig. 4c) by the presence of hexagonal arrays of $c a .5 \mathrm{~nm}$ wide parallel channels. In $\mathrm{N}_{2}$ adsorption these channels are responsible for an hysteresis loop with steep parallel branches (Fig. 5b) characteristic of capillary condensation in open ended cylindrical mesopores. ${ }^{11,12}$

The formation mechanism of COK-12 is known in detail. It proceeds via coalescence of individual silica-coated spherical

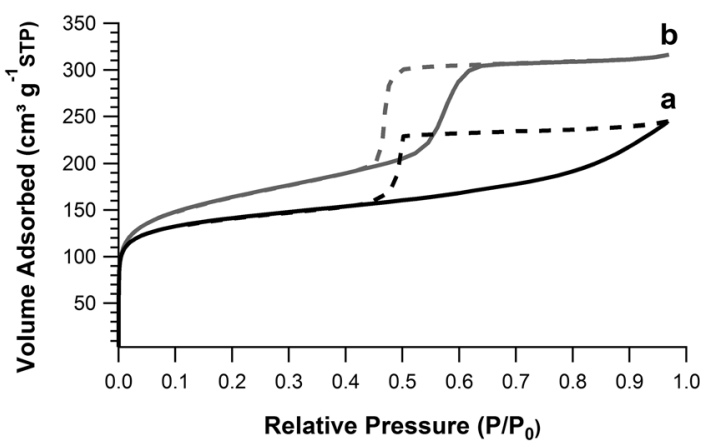

Fig. 5 Nitrogen sorption isotherm of FPS (a) and ordered mesoporous silica, COK-12 (b) calcined at $550{ }^{\circ} \mathrm{C}$. Isotherm (a) is characteristic for a material with pores larger than $50 \mathrm{~nm}$. The strong hysteresis at partial pressure $P / P_{0}=$ 0.45 is indicative for narrow openings to these macropores. Isotherm (b) displays ordered cylindrical mesopores of approximately $5 \mathrm{~nm}$. 
micelles to form cylinders, which assemble in hexagonal arrays. ${ }^{12}$ Addition of flurbiprofen to the synthesis mixture causes strong swelling of the spherical micelles from ca. $14 \mathrm{~nm}$ to $180 \mathrm{~nm}$ (Fig. 1). Silica is precipitated on the surface of these extra-large micelles upon addition of sodium silicate solution. The FPS material corresponds to an orderless packing of silica coated swollen micelles spheres occluding flurbiprofen-bearing P123 micelles according to SAXS (Fig. 4). The nitrogen adsorption isotherm (Fig. 5) is in agreement with formation of large voids with microporous windows. Calcination causes local damage and formation of holes in the silica skin of the particles through which the gaseous degradation products from P123 and flurbiprofen can be evacuated.

\section{Location and physical state of flurbiprofen in FPS}

In DSC analysis, crystalline flurbiprofen displays a melting peak at $115.6{ }^{\circ} \mathrm{C}$ (Fig. 6). The thermogram of FPS did not show any signal related to melting of crystalline flurbiprofen, suggesting flurbiprofen was molecularly dispersed or present in amorphous state.

To further distinguish between crystalline, amorphous and dispersed state, ${ }^{13} \mathrm{C}$ single-pulse and ${ }^{1} \mathrm{H} \rightarrow{ }^{13} \mathrm{C}$ CPMAS NMR spectra of FPS were recorded (Fig. 7). CPMAS transfer relies on ${ }^{1} \mathrm{H}-{ }^{13} \mathrm{C}$ dipolar interaction, which is averaged out to zero for mobile moieties. On the contrary, in single pulse experiments with short relaxation delay, only the mobile species are detected. Crystalline species have a much longer longitudinal relaxation time $T_{1}$ values, and are not visible on the spectrum. Therefore, using the combination of these NMR techniques, it was possible to unambiguously distinguish between flurbiprofen within the P123 micelles (detected with single-pulse spectra; Fig. 7a and c) and extraneous flurbiprofen crystallized outside the micelles (detected with CPMAS NMR; Fig. 7b and d), without special sample manipulation. Quantitative analysis of the ${ }^{13} \mathrm{C}$ single-pulse NMR spectra is done by integrating the ${ }^{13} \mathrm{C}$ resonances $($ e.g., that at $175 \mathrm{ppm}$, which corresponds to the $\mathrm{C}=\mathrm{O}$ from the flurbiprofen in the inclusion and the two narrow signals at 75-76 ppm which are the $\mathrm{CH}_{2}-\mathrm{O}$ of the pPO moieties, and taking into account the 70 pPO motives in the P123 as mentioned in the experimental section). These results indicate that a maximum 1:17 ratio between flurbiprofen molecules

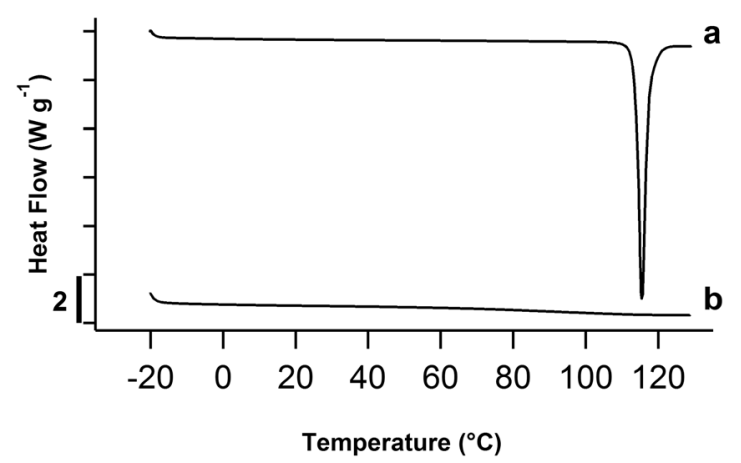

Fig. 6 DSC thermograms of (a) crystalline flurbiprofen and (b) FPS. Results are represented endotherm down.

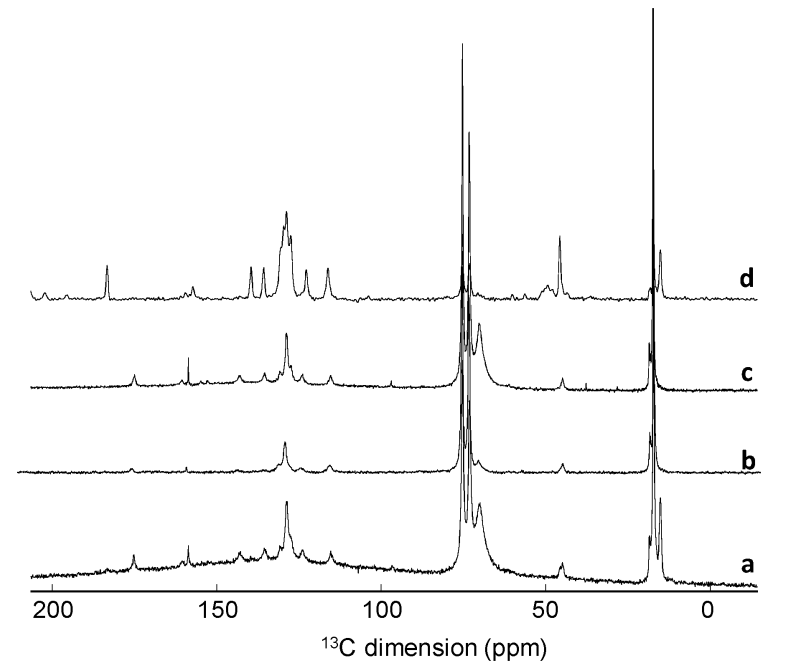

Fig. $7{ }^{13} \mathrm{C}$ single-pulse MAS NMR spectra of FPS (a) and a material with 4 times higher flurbiprofen content (c). Only flurbiprofen solubilized within micelles is detected. ${ }^{13} \mathrm{C}$ CPMAS NMR spectrum of FPS (b) and material with 4 times higher loading (d). This spectrum reveals the presence of crystalline flurbiprofen.

and PO units is observed, in agreement with the ratio yielded by the molar synthesis composition $(1: 19)$. Only when an excess amount of flurbiprofen was added during synthesis, corresponding to 4 times the nominal amount, the presence of crystalline flurbiprofen was detected using CPMAS.

\section{In vitro release of flurbiprofen from FPS}

Release of flurbiprofen from FPS was investigated in phosphate buffer (0.1 M, pH 6.8). Under the experimental conditions release of the entire dose corresponded to a flurbiprofen concentration of $120 \mathrm{mg}$ flurbiprofen $\mathrm{l}^{-1}$. In phosphate buffer the solubility of flurbiprofen is about $2800 \mathrm{mg} \mathrm{l}^{-1}$ such that sink conditions were established. ${ }^{14,15}$ Flurbiprofen release was fast and quantitative (Fig. 9). In the phosphate medium at pH 6.8 the carboxylic acid function of flurbiprofen molecule is deprotonated, which increases solubility. The release of flurbiprofen from FPS was confirmed by ${ }^{1} \mathrm{H}$ and ${ }^{13} \mathrm{C}$ solid-state NMR of recovered solid material after $4 \mathrm{~h}$. Signals of the flurbiprofen molecules were no longer present and only P123 remained (Fig. 10). TGA analysis revealed that about $40 \%$ of the Pluronic was released in the dissolution medium in $4 \mathrm{~h}$ (see ESI, $\dagger$ Fig. S5).

${ }^{13} \mathrm{C}$ NMR resonance frequencies of flurbiprofen in FPS were different from the ones of crystalline flurbiprofen. A closer analysis showed that only the carbon atoms close to the carbonyl function (lines labeled a, d, j, k and l) significantly shifted position (Fig. 8). The crystalline form of flurbiprofen is made of dimers, linked together through hydrogen bonding between two neighboring carboxyl groups. ${ }^{30,31}$ The modified chemical shifts confirmed dimers of flurbiprofen no longer existed once flurbiprofen was solubilized in the P123 micelles, which further indicated that the flurbiprofen molecules are well dispersed and isolated in the micelles.

In simulated gastric fluid $(\mathrm{pH}$ 1.2) flurbiprofen release was much more limited (Fig. 9). The solubility limit of ca. $6 \mathrm{mg} \mathrm{l}^{-1}$ 


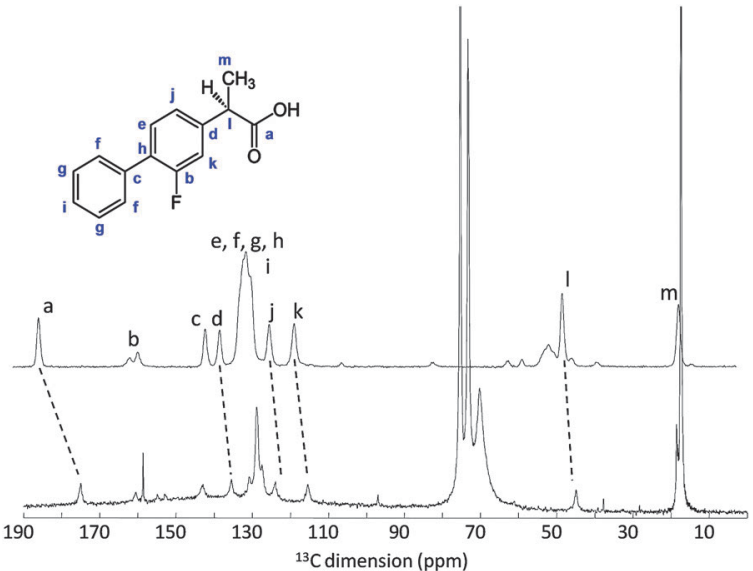

Fig. $8{ }^{13} \mathrm{C}$ single-pulse MAS NMR and ${ }^{13} \mathrm{C}$ CPMAS NMR spectrum of FPS The molecule of flurbiprofen, with labeled carbon atoms, is shown as inset. Assignment is made based on previously reported first-principle calculations. ${ }^{32}$ Dash lines indicate the signals that shift depending on the physical state of flurbiprofen.

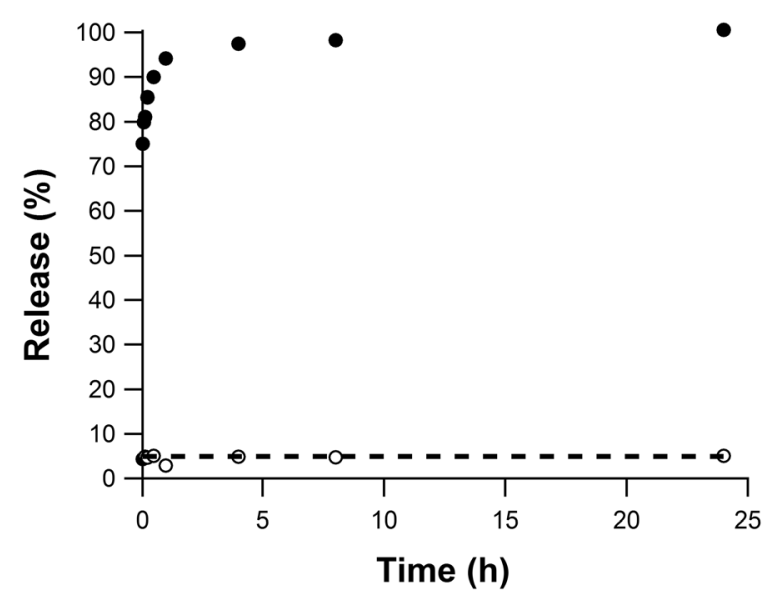

Fig. 9 Release of flurbiprofen form FPS in $(O)$ simulated gastric fluid (0.1 M, pH 1.2) and (1) phosphate buffer $(0.1 \mathrm{M}, \mathrm{pH}$ 6.8) at $120 \mathrm{mg}$ flurbiprofen $\mathrm{I}^{-1}$ dosage. The dashed line represents solubility of flurbiprofen in SFG solution (6 $\left.\mathrm{mg} \mathrm{l}^{-1}\right)$

was reached within minutes. Only about $5 \%$ of the introduced dose was released. The retention of the majority of the flurbiprofen in the FPS was confirmed by ${ }^{1} \mathrm{H}$ and ${ }^{13} \mathrm{C}$ solid-state NMR spectra of the FPS solids recovered after $4 \mathrm{~h}$ (Fig. 10, see ESI, $\dagger$ Fig. S3 and S5). Interestingly, $2 \mathrm{D}{ }^{1} \mathrm{H}^{1}{ }^{1} \mathrm{H}$ NMR revealed flurbiprofen remained solubilized in the pPO core of the P123 micelles (see ESI, $\dagger$ Fig. S4).

A final release experiment simulated the gastric-to-intestinal transition by examining release in SGF, followed by release in the phosphate buffered media (Fig. 11). In SGF, release was limited to $5.6 \%$ or $6 \mathrm{mg}^{-1}$. Transfer to the phosphate buffered medium released more than $90 \%$ of flurbiprofen within 20 minutes.

The drug delivery principle of FPS capsules is different from OMS materials. Surfactant present inside the material also can influence the release behavior. In general, the fast burst release of flurbiprofen closely resembles the release profile of other

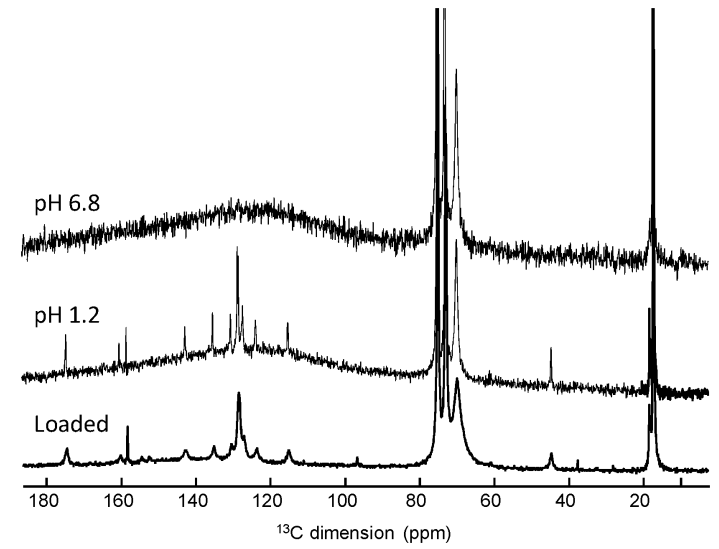

Fig. $10{ }^{13} \mathrm{C}$ single-pulse MAS NMR spectra of FPS initial sample, and of the same sample after flurbiprofen release experiments at $\mathrm{pH} 1.2$ and 6.8 .

poorly soluble, weak acidic drug molecules loaded into SBA-15 silica materials via incipient wetness loading procedures. ${ }^{13,33}$ Active compounds loaded into evacuated pores typically adsorb on the silica surface mainly through hydrogen bonds with silanol groups present on the surface and are released via competitive adsorption of water. As water molecules enter the pores, water preferentially interacts with the surface silanols, displacing the drug molecules. These drug molecules desorb from the surface and are released from inside the porous structure. ${ }^{13}$ Classically loaded indomethacin and glibenclamide showed similar differences in release behavior between SGF and simulated intestinal fluid. ${ }^{33}$ Discrepancies in the release of these drug compounds in SGF were partially ascribed to charge interactions between the silica surface and the active compounds. Under acidic conditions, the molecules are not ionized, while the silica surface has a net positive charge, whereas at neutral $\mathrm{pH}$ and in simulated intestinal fluid both the drug molecules and the silica surface carry negative charges.

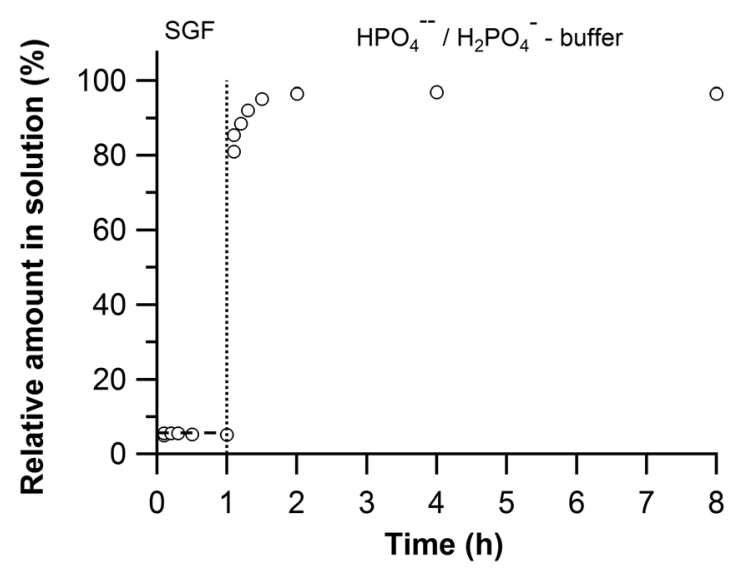

Fig. 11 Release of flurbiprofen from FPS, simulating the gastro-intestinal tract. After 60 minutes, aliquots were transferred from a $0.1 \mathrm{M} \mathrm{HCl}$ to a $0.1 \mathrm{M}$ phosphate buffered solution $(\mathrm{pH}$ 6.8). $100 \%$ corresponds to a concentration of $106.6 \mathrm{mg} \mathrm{l}^{-1}$ in SGF and $10.66 \mathrm{mg} \mathrm{l}^{-1}$ in phosphate buffer. The horizontal dashed line represents the equilibrium solubility of flurbiprofen in SGF $\left(6 \mathrm{mg} \mathrm{l}^{-1}\right)$. 
Charge repulsion will likely promote desorption and subsequently release, while under acidic conditions drug molecules remain embedded in the porous silica structure. ${ }^{33}$ A similar explanation for the release mechanism can be applied here and is consolidated by the stabilization of flurbiprofen inside the hydrophobic pPO core of the P123 template at low $\mathrm{pH}$ and release from the surfactant micelles due to ionization at more neutral pH. The non release in SGF and inability to wash out the surfactant template thus reflects the inability of water to enter the silica capsules and effectively displace the surfactant and the drug molecules.

\section{Conclusions}

Flurbiprofen is incorporated in non-ionic, non-toxic triblock copolymer which forms spherical micelles, keeping the hydrophobic drug compound inside the hydrophobic core of the surfactant micelle. Addition of sodium silicate at neutral $\mathrm{pH}$ and room temperature leads to spontaneous formation of 200-400 nm capsules filled with flurbiprofen loaded surfactant assemblies. Flurbiprofen was loaded into a mesoporous structure without the need to first evacuate the pores, thereby avoiding additional loading steps. Characterization with DCS, ${ }^{13} \mathrm{C}$ and $2 \mathrm{D}{ }^{1} \mathrm{H}-{ }^{1} \mathrm{H}$ NMR spectroscopy shows flurbiprofen is molecularly dispersed in the hydrophobic core of the pPO micelles.

Release of flurbiprofen from the carrier material was controlled by $\mathrm{pH}$. Under acidic conditions, release is restricted due to low solubility in the medium as well as enhanced hydrophobic interactions between flurbiprofen and PO chains of the triblock copolymer assembly. At $\mathrm{pH}$ 6.8, characteristic of intestinal fluid, flurbiprofen is deprotonated thus becoming more hydrophilic, releasing from the hydrophobic environment of the surfactant core and the carrier material.

This easy synthesis routine to load flurbiprofen in an inorganic carrier material, solubilized in the surfactant template can possibly be extended to other hydrophobic molecules. Hydrophobic drug molecules are solubilized inside the hydrophobic core of Pluronic P123 and directly immobilized inside the pores of the inorganic silica capsule during material formation. This new synthesis approach, using the citrate silicate synthesis procedure, does not require any organic solvents and can be performed under physiological conditions of room temperature and neutral $\mathrm{pH}$, making this a suitable method for the incorporation of sensitive hydrophobic molecules inside porous silica carriers.

\section{Acknowledgements}

JAM acknowledges the Flemish government for long-term structural funding (Methusalem). CM is grateful to the French ANR for financial support. Florent Baudouin is acknowledged for help in recording the NMR data. We gratefully thank Monica Vialpando for performing DSC measurements. We also would like to thank Patrick Rombouts and Randy Mellaerts for their assistance with the release experiments.

\section{References}

1 C. A. Lipinski, J. Pharmacol. Toxicol. Methods, 2000, 44, 235-249.

2 R. Mellaerts, R. Mols, P. Kayaert, P. Annaert, J. Van Humbeeck, G. Van den Mooter, J. A. Martens and P. Augustijns, Int. J. Pharm., 2008, 357, 169-179.

3 C.-H. Tsai, J. L. Vivero-Escoto, I. I. Slowing, I. J. Fang, B. G. Trewyn and V. S. Y. Lin, Biomaterials, 2011, 32, 6234-6244.

4 M. Van Speybroeck, V. Barillaro, T. Do Thi, R. Mellaerts, J. Martens, J. Van Humbeeck, J. Vermant, P. Annaert, G. Van Den Mooter and P. Augustijns, J. Pharm. Sci., 2009, 98, 2648-2658.

5 P. Botella, A. Corma and M. Quesada, J. Mater. Chem., 2012, 22, 6394-6401.

6 Q. He, J. Shi, F. Chen, M. Zhu and L. Zhang, Biomaterials, 2010, 31, 3335-3346.

7 Y.-H. Son, M. park, Y. B. Choy, H. R. Choi, D. S. Kim, K. C. Park and J.-H. Choy, Chem. Commun., 2007, 2799-2801.

8 K. M. Tyner, S. R. Schiffman and E. P. Giannelis, J. Controlled Release, 2004, 95, 501-514.

9 N. W. Clifford, K. S. Iyer and C. L. Raston, J. Mater. Chem., 2008, 18, 162-165.

10 N. Vlachy, D. Touraud, J. Heilmann and W. Kunz, Colloids Surf., B, 2009, 70, 278-280.

11 J. Jammaer, A. Aerts, J. D'Haen, S. Jin Won and J. A. Martens, J. Mater. Chem., 2009, 8290-8293.

12 J. Jammaer, T. S. van Erp, A. Aerts, C. E. A. Kirschhock and J. A. Martens, J. Am. Chem. Soc., 2011, 133, 13737-13745.

13 R. Mellaerts, J. A. G. Jammaer, M. Van Speybroeck, H. Chen, J. Van Humbeeck, P. Augustijns, G. Van den Mooter and J. A. Martens, Langmuir, 2008, 24, 8651-8659.

14 S. Alexander, W. de Vos, T. Castle, T. Cosgrove and S. Prescott, Langmuir, 2012, 28, 6539-6545.

15 S. Chandran, A. Roy and R. N. Saha, AAPS PharmSciTech, 2008, 9, 1031-1037.

16 A. Avdeef, C. M. Berger and C. Brownell, Pharm. Res., 2000, 17, 85-89.

17 B. M. Fung, A. K. Khitrin and K. Ermolaev, J. Magn. Reson., 2000, 142, 97-101.

18 D. J. States, R. A. Haberkorn and D. J. Ruben, J. Magn. Reson., 1982, 48, 286-292.

19 D. Massiot, F. Fayon, M. Capron, I. King, S. Le Calvé, B. Alonso, J.-O. Durand, B. Bujoli, Z. Gan and G. Hoatson, Magn. Reson. Chem., 2002, 40, 70-76.

20 S. Manet, A. Lecchi, M. Imperor-Clerc, V. Zholobenko, D. Durand, C. L. P. Oliveira, J. S. Pedersen, I. Grillo, F. Meneau and C. Rochas, J. Phys. Chem. B, 2011, 115, 11318-11329.

21 R. Ganguly, V. K. Aswal and P. A. Hassan, J. Colloid Interface Sci., 2007, 315, 693-700.

22 P. Alexandridis, J. F. Holzwarth and T. A. Hatton, Macromolecules, 1994, 27, 2414-2425.

23 R. Basak and R. Bandyopadhyay, Langmuir, 2013, 29, 4350-4356. 
24 E. V. Batrakova, T. Y. Dorodnych, E. Y. Klinskii, E. N. Kliushnenkova, O. B. Shemchukova, O. N. Goncharova, S. A. Arjakov, V. Y. Alakhov and A. V. Kabanov, Br. J. Cancer, 1996, 74, 1545-1552.

25 E. V. Batrakova and A. V. Kabanov, J. Controlled Release, 2008, 130, 98-106.

26 D. Y. Zhao, J. L. Feng, Q. S. Huo, N. Melosh, G. H. Fredrickson, B. F. Chmelka and G. D. Stucky, Science, 1998, 279, 548-552.

27 L. Cao and M. Kruk, RSC Adv., 2014, 4, 331-339.

28 M. Kruk and C. M. Hui, Microporous Mesoporous Mater., 2008, 114, 64-73.
29 Z. Liu, D. Liu, L. Wang, J. Zhang and N. Zhang, Int. J. Mol. Sci., 2011, 12, 1684-1696.

30 J. L. Flippen and R. D. Gilardi, Acta Crystallogr., Sect. B, 1975, 31, 926-928.

31 A. L. Grzesiak and A. J. Matzger, J. Pharm. Sci., 2007, 96, 2978-2986.

32 J. R. Yates, S. E. Dobbins, C. J. Pickard, F. Mauri, P. Y. Ghi and R. K. Harris, Phys. Chem. Chem. Phys., 2005, 7, 1402-1407.

33 M. van Speybroeck, R. Mellaerts, T. Thao Do, J. A. Martens, J. Van Humbeeck, P. Annaert, G. Van den Mooter and P. Augustijns, J. Pharm. Sci., 2011, 100, 4864-4876. 